\title{
LA ISLA DE CALOR EN LA CIUDAD DE LLORET DE MAR
}

\author{
María del Carmen Moreno García ${ }^{1}$ \\ Juan Antonio Serra Pardo \\ ${ }^{1}$ Grupo de Climatología. Departamento de Geografía Física y Análisis Regional de la Universidad de Barcelona \\ mcmoreno@ub.edu, juanantonioserrapardo@gmail.com
}

\section{RESUMEN}

Este artículo muestra el resultado de un trabajo de investigación sobre el fenómeno de la isla de calor en la ciudad de Lloret de Mar, donde se evidencia la existencia del fenómeno y se caracteriza. Utilizando el método de los transectos se han realizado mediciones de temperatura en cuatro campañas anuales entre julio del 2010 y marzo de 2014, durante el verano e invierno. El análisis de los 11.360 valores térmicos recopilados muestra una isla de calor urbana con una intensidad media de $3,3^{\circ} \mathrm{C}$, mientras que la intensidad máxima alcanza los $6,6^{\circ} \mathrm{C}$. El centro de la isla se sitúa sobre el sector litoral de la ciudad y parte del barrio del Nucli Antic. Se presentan además algunas diferencias verano-invierno observadas.

Palabras clave: clima urbano, isla de calor urbano, contraste térmico urbano-rural, tansectos urbanos, Lloret de Mar, diferencias verano-invierno.

\section{ABSTRACT}

This article shows the result of a research based on heat island phenomenon in the city of Lloret de Mar, demonstrating that the existence of the phenomenon and characterizing it. Using the urban transects method, four annuals campaigns of temperature capture were realized from July of 2010 until March 2014, during summers and winters. The analysis of the 11.360 thermal values shows that the average intensity of the urban heat island is

Fecha de recepción: marzo 2015.

Fecha de aceptación: diciembre 2015. 
$3,3^{\circ} \mathrm{C}$, while the maximum intensity reaches $6,6^{\circ} \mathrm{C}$. The core is placed on the coastline and Nucli Antic neighborhood. Morever, it presents some summer-winter differences that have been observed.

Keywords: urban climate, urban heat island, urban-rural contrast thermal, urban transects, Lloret de Mar, summer-winter differences.

\section{INTRODUCCIÓN}

El geógrafo español Manuel de Terán dijo que la ciudad representa "la forma más radical de transformación del paisaje natural, pues su impacto no se limita a cambiar la morfología del terreno, nuevas construcciones, otro plano y disposición del territorio, ni tampoco la aglomeración humana o mecánica que determina, sino que todo ello modifica las mismas condiciones climáticas y ambientales, elevando la temperatura y afectando al régimen de precipitaciones y de vientos" (Tornero, Pérez y Gómez, 2006).

A lo largo del siglo XX es cuando se ha ido conformando una abundante bibliografía entorno al clima de las ciudades, que demuestra el interés de Terán y otros investigadores por conocer, en general, la influencia del hombre en el medio ambiente, y, en particular, las alteraciones o modificaciones climáticas antropogénicas en las ciudades.

No obstante, la percepción sensorial acerca del clima urbano se encuentra en la antigüedad. Un claro ejemplo es Hipócrates, quién con sus "Aforismos" y "De los aires, de las aguas y de los lugares" presenta la influencia que el medio tiene sobre el hombre, especialmente en su salud y bienestar. Otro ejemplo está en Aristóteles o Vitrubio para quienes la construcción de las ciudades debía tener en cuenta la presencia del viento y la aireación. Por no hablar de la cultura popular que durante años ha ido emplazando las viviendas bajo criterios bioclimáticos (orientación hacia el sur y no al norte) en busca de una mejor confortabilidad.

La magnitud del incremento de la urbanización parece haber convertido a las ciudades en ideales laboratorios de experimentación de los efectos derivados de la acción humana sobre el medio natural, de sus impactos sobre la sociedad y de las medidas de adaptación y mitigación necesarias para combatirlos (Fernández, 2009).

Esto ha generado, en los últimos años, una gran cantidad de estudios realizados sobre clima urbano, una gran parte recogidos por Arnfield (2003), fundamentados en la transcendencia que tiene sobre el confort y la salud de la población que habitan en las ciudades. Cada día más, las administraciones locales tratan de realizar una gestión ambiental dirigida a controlar y mejorar la calidad ambiental de un entorno cada vez más modificado.

Una de las modificaciones del clima urbano que mejor se conoce es el fenómeno de la isla de calor o UHI (Urban Heat Island), que ha sido muy estudiado, especialmente en Norteamérica y países asiáticos. Se trata de un fenómeno de origen térmico que se desarrolla en las áreas urbanas causado por las diferencias de temperatura existentes entre el centro de la ciudad y la de sus alrededores. El contraste térmico entre lo urbano y lo rural, se debe principalmente a tres factores: la producción directa de calor por combustión, el desprendimiento gradual del calor almacenado durante el día en las construcciones de 
ladrillo, hormigón y demás materiales similares y la radiación que es devuelta a la superficie terrestre por reflexión en la capa de impurezas atmosféricas (Domínguez, 2002).

Se pueden distinguir dos tipos de isla de calor: la primera, denominada isla de calor atmosférica, señala las diferencias térmicas entre el aire de las zonas urbanas y de las rurales; la segunda, denominada isla de calor superficial, representa la diferencia de la temperatura registrada en los distintos materiales y superficies urbanas (pavimentos, aceras, tejados de los edificios, etc.). Entre ambas se pueden observar diferencias en los mecanismos de formación, las técnicas empleadas para su identificación y las medidas de mitigación (Fernández, 2009).

El estudio que se presenta en este artículo, analiza la influencia de la urbanización en la temperatura para el caso de una ciudad de tamaño medio, describiendo la configuración de su isla de calor y su localización dentro de la ciudad. Dos son los objetivos principales:

a) Ampliar las investigaciones sobre clima urbano que comienzan a desarrollarse en España a partir de los años 80 del siglo XX, basadas en el estudio de las características térmicas de diferentes ciudades españolas (López et al, 1993; Fernández et al, 1998; Brunet, 1992; Dorta, Marzol y Rodriguez, 1992; Cuadrat, De La Riva y López, 1993; Moreno, 1993; Bello, 1994 y 1995; Ortigosa, Sobrón y Gómez et al, 1998; Montávez, Rodríguez y Jiménez, 2000; Alonso, Labajo y Fidalgo 2003; Serrano, Cuadrat, Sanz, 2005; Castillo, 2006; Ramiro, 2006; Ruíz, Romero, Mayer y Hernández, 2008; De la Morena, 2010).

b) Proseguir con el estudio del fenómeno de la isla de calor en ciudades turísticas a partir de la comparación térmica del periodo de máxima actividad (verano) y de menor actividad (invierno), observando si la actividad turística introduce modificaciones en las principales características del fenómeno (Serra, 2007).

\section{II. ÁREA DE ESTUDIO}

La ciudad de Lloret de Mar se encuentra situada (figura 1) en las coordenadas geográficas $41^{\circ} 42^{\prime}$ de latitud norte; $2^{\circ} 50^{\prime}$ de longitud este. En el extremo nororiental de la Península Ibérica, en Cataluña, este municipio de la provincia de Gerona, forma parte de la Comarca de la Selva y de la Costa Brava. Con una población de 38.624 habitantes y una superficie de $48,7 \mathrm{~km}^{2}$, en la franja litoral, limita con los municipios de Blanes, por el suroeste, y de Tossa de Mar, por el noreste, mientras que en el interior limita con Vidreres y Maçanet de la Selva, por el norte, y con Tordera, por el noroeste.

Aunque los primeros asentamientos humanos que existieron en el municipio que hoy ocupa Lloret de Mar se encontraron el siglo III aC, en el Puig del Castellet y en Montbarbat, no es hasta el siglo XIV cuando se acaba formando el núcleo original que da lugar a Lloret de Mar.

El emplazamiento del núcleo original del la ciudad, extendiéndose enfrente de la playa de Lloret, muestra una ciudad que ha mantenido una intensa relación con el mar. Una relación que tendrá su máximo esplendor con el comercio marítimo con las colonias de América que generó una importante actividad económica de astilleros y de intercambio. Esta actividad económica permitirá acumular fortunas a varios lloretenses, quienes serán los que comiencen a impulsar la transformación urbana de la villa y su crecimiento. Una vez se 
pierden las colonias, este dinamismo también se pierde y no vuelve a recuperarse hasta que llegan los turistas atraídos por la playa de Lloret. Unos turistas, que en primera instancia proceden de Barcelona, que poco a poco se irán internacionalizando debido al importante trabajo de promoción turística realizado desde la localidad y al boom turístico.
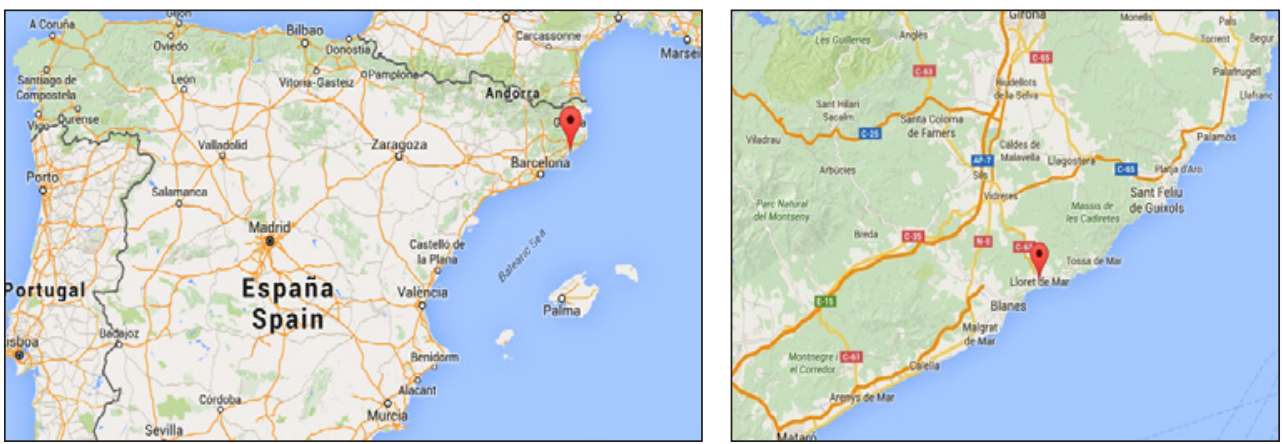

Fuente: Google Maps.

Conviene destacar que Lloret de Mar fue pionera en el turismo europeo de los años 50, convirtiéndose en una de los destinos turísticos más importantes de España. La actividad turística ha sido, precisamente, uno de los factores que ha generado las transformaciones territoriales más agresivas del municipio. El boom turístico de los 60 significó un cambio cualitativo en el uso del suelo, en muchos casos, sin planificación. Bajo la influencia de esta actividad se ha ido urbanizando el territorio, consumiendo los espacios libres del casco urbano, colmatándolo y ocupando nuevos, ya sea en forma de nuevos barrios o de urbanizaciones, siguiendo esquemas de ciudad-jardín.

De esta manera, la morfología urbana que presenta Lloret de Mar en la actualidad viene caracterizada, en gran medida, por el enorme impacto que ha generado el turismo. Un impacto que ha provocado un rápido crecimiento de la ciudad que en pocos años ha pasado de ser un pueblo turístico de costa a ser una ciudad turística. Este cambio, de pueblo a ciudad, queda bien ejemplificado en la evolución demográfica que ha experimentado en los últimos años, caracterizada por un incremento sustantivo de su población (figura 2). Dicho crecimiento ha sido posible gracias a una inmigración nacional (sur y suroeste de España), que ha generado barrios periféricos sin planificación previa y carentes de infraestructuras y/o servicios urbanos básicos, debido a la necesidad de dar una rápida respuesta a la llegada de estos nuevos residentes.

La estructura urbana del municipio de Lloret de Mar queda definida, a grandes rasgos, por cinco unidades (figura 3):

1. El Casco antiguo. En este sector están el barrio del Nucli Antic y el barrio de la Riera. Este es el sector donde se fundó la ciudad y donde se concentró la población de Lloret hasta los años sesenta. Muestra una urbanización compacta con una elevada densidad 
edificatoria y, por tanto, escasos espacios libres. También presenta una elevada densidad residencial, aproximadamente de $140 \mathrm{hab} / \mathrm{Ha}$. Las calles son estrechas y una gran parte son peatonales. Aquí se concentra 2/3 partes de los servicios y de la oferta turística del municipio, incluyendo gran parte de los equipamientos municipales educativos, sociales, sanitarios y de la administración pública, entidades bancarias, gran parte del comercio (especialmente en los sectores peatonales), hoteles, bares, restaurantes, discotecas...

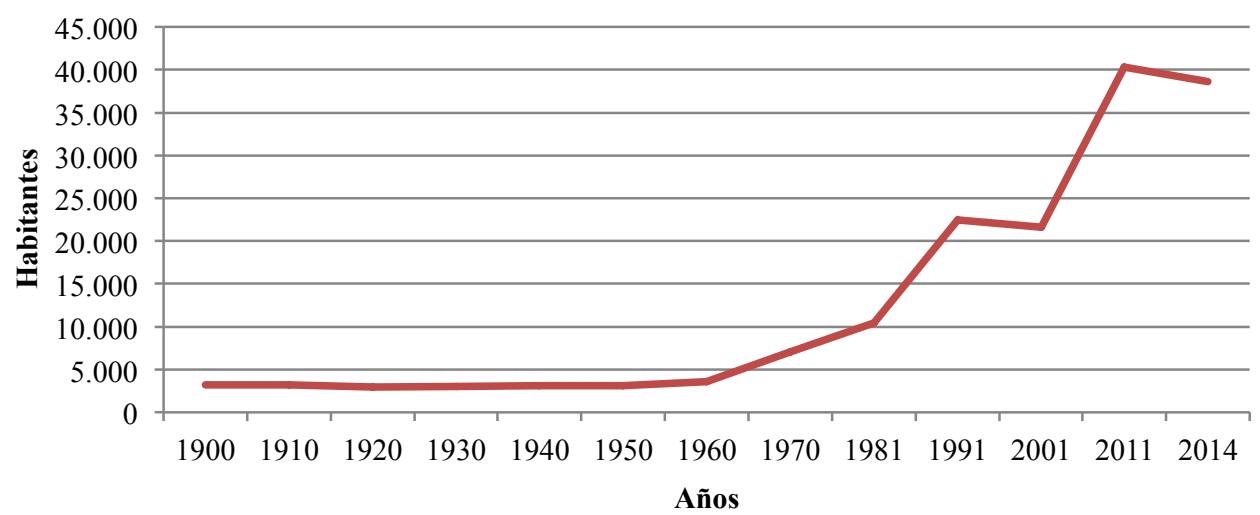

Fuente: elaboración propia a partir de datos del INE.

2. Barrios residenciales periféricos. Los barrios que conforman esta unidad son los de Mas Arboç-Sant Quirze, El Rieral, Puigventós, Can Sabata, Can Carbó, El Moli, Can Ribalaigua, Mas Vila, Mas Ballell, Can Ballell, barrio de Pescadors, Cala Banys y Can Coll. Dentro de esta unidad están presentes los barrios periféricos al centro antiguo, alguno de los cuales se desarrollaron sin una planificación adecuada y con un déficit importante de servicios. Así pues, se trata de barrios de "reciente" construcción que se ubican en el perímetro del casco antiguo. Presentan una alta densidad residencial, pero una baja actividad terciaria. Estos barrios presentan una urbanización más moderna con una trama más cuadriculada y unas calles más anchas. Aquí hay presencia de algún hotel. Esta estructura comprende todos los sectores de expansión de la ciudad desde los años 70. No obstante, también se pueden encontrar sectores de baja densidad residencial y una topografía con fuertes pendientes como los de Can Coll y Cala Banys, o simplemente con una baja densidad residencial como Sant Quirze.

3. Fenals. Este sector, coincidente con el barrio del mismo nombre, es el segundo más poblado y el que mayor extensión tiene. Presenta una mezcla de usos entre residencial (principal y secundaria), turísticos (destinado a un sector más familiar) y comercial, que no se da en ninguna otra área de la ciudad. Este amplio sector se encuentra un 
poco desconectado del casco antiguo debido a la orografía, ya que el monte del sector del Castell le separa de él. Este hecho, junto con la presencia de la playa, ha provocado un crecimiento urbano independiente al del resto al casco antiguo. En la zona más próxima a la playa se dan unos usos característicos de zona turística, con la presencia de grandes hoteles, edificaciones residenciales (segunda residencia), bares, restaurantes y tiendas de souvenirs; hacia el interior, cerca de la avenida vila de Blanes se dan usos más residenciales, con edificios de nueva construcción y poca actividad terciaria. Esta avenida, una de las principales vías de entrada a la ciudad, actúa como atractora de superficies comerciales (Dia, Consum, Eroski) y gasolineras. Por último, cabe mencionar que este sector presenta una trama regular con la presencia de algunas avenidas y calles anchas.

4. Zona industrial. A las afueras de Lloret, en la carretera C-63 que va a Vidreres, se encuentra un pequeño sector que coincide con las características de polígono industrial. Se trata de la única área de la ciudad que presenta esta particularidad.

5. Las urbanizaciones. En el municipio de Lloret existen numerosas urbanizaciones dispersas por el término municipal. Éstas significan 1/3 de la superficie urbanizada del municipio. Se trata de pequeñas áreas urbanas de baja densidad, mayormente destinadas a segunda residencia y con importantes déficits de servicios. Las principales urbanizaciones son: Santa Clotilde, Condado del Jaruco, Roca Grossa, la Montgoda, Lloret Verd, Lloret Residencial, La Creu de Lloret, Lloret Blau, Els Pinars, Turo de Lloret, Mas Romeu Residencial.

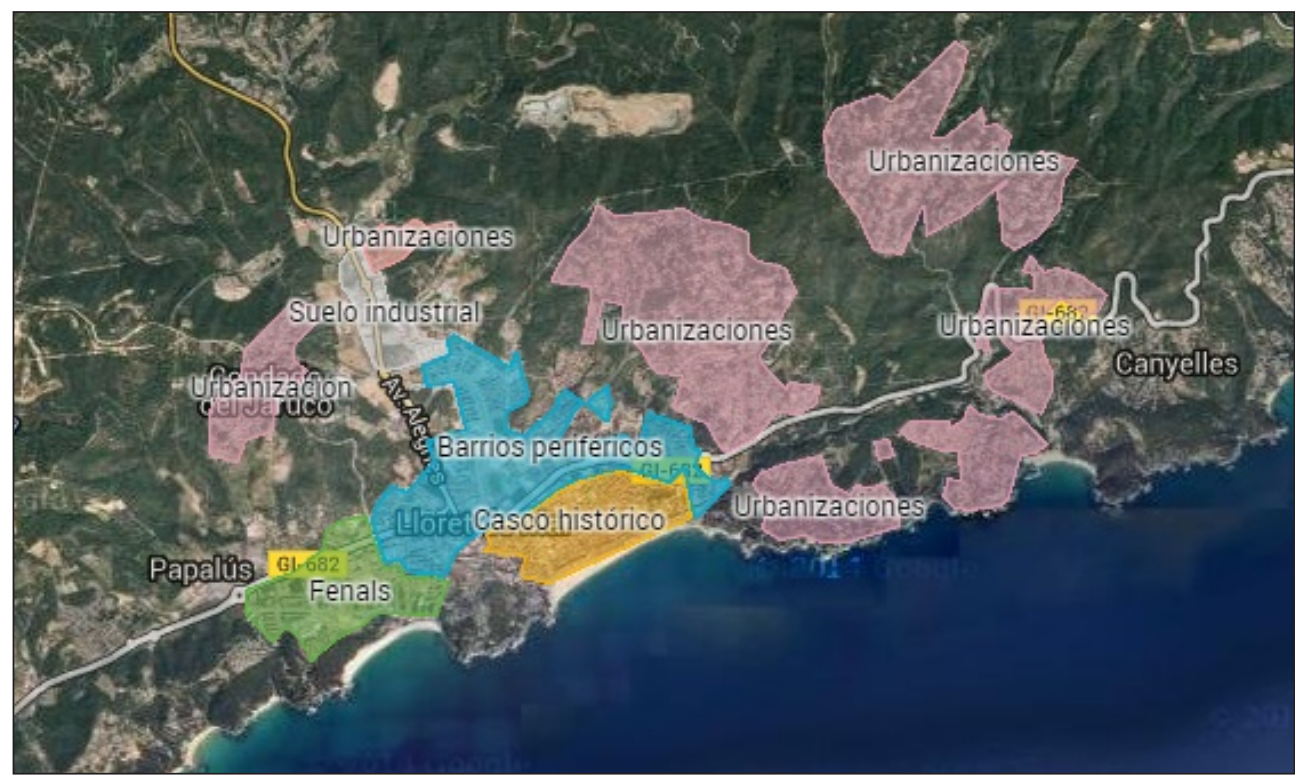

Fuente: elaboración propia a partir de Google Maps. 


\section{METODOLOGÍA}

La metodología empleada se basa en la técnica de los transectos, consistente en la utilización de un vehículo automóvil equipado con un temrohigrómetro digital que permite obtener unas series de medidas meteorológicas sobre el terreno en diversos puntos a lo largo de un recorrido. El equipo instrumental, de baja inercia, constaba de una sonda de ambiente que fue ubicaba en el exterior del vehículo.

Los transectos diseñados fueron tres, presentando una longitud total de $18,9 \mathrm{~km}$ (figura $3)$. Entre los tres transectos, se recopilaban un total de 71 valores térmicos diarios a través de los 47 puntos de observación distribuidos a lo largo de los mismos. Hay que indicar la existencia de puntos comunes entre los transectos para permitir un ajuste o corrección en las temperaturas anotadas y, así, poder ser consideradas como simultáneas.

El transecto más importante es el número 3, ya que es el que mayor extensión presenta y el que atraviesa el sector más densamente edificado del núcleo urbano. Éste es el utilizado para comprobar la simultaneidad de las temperaturas debido a su importancia, al ser el que más puntos en común presenta con los otros dos y al ser el último que se realizaba, habiendo transcurrido mayor tiempo desde la puesta de sol (2/3h). A continuación se pasan a presentar los transectos (figura 4).

Figura 4

\section{RECORRIDO DE LOS TRANSECTOS}

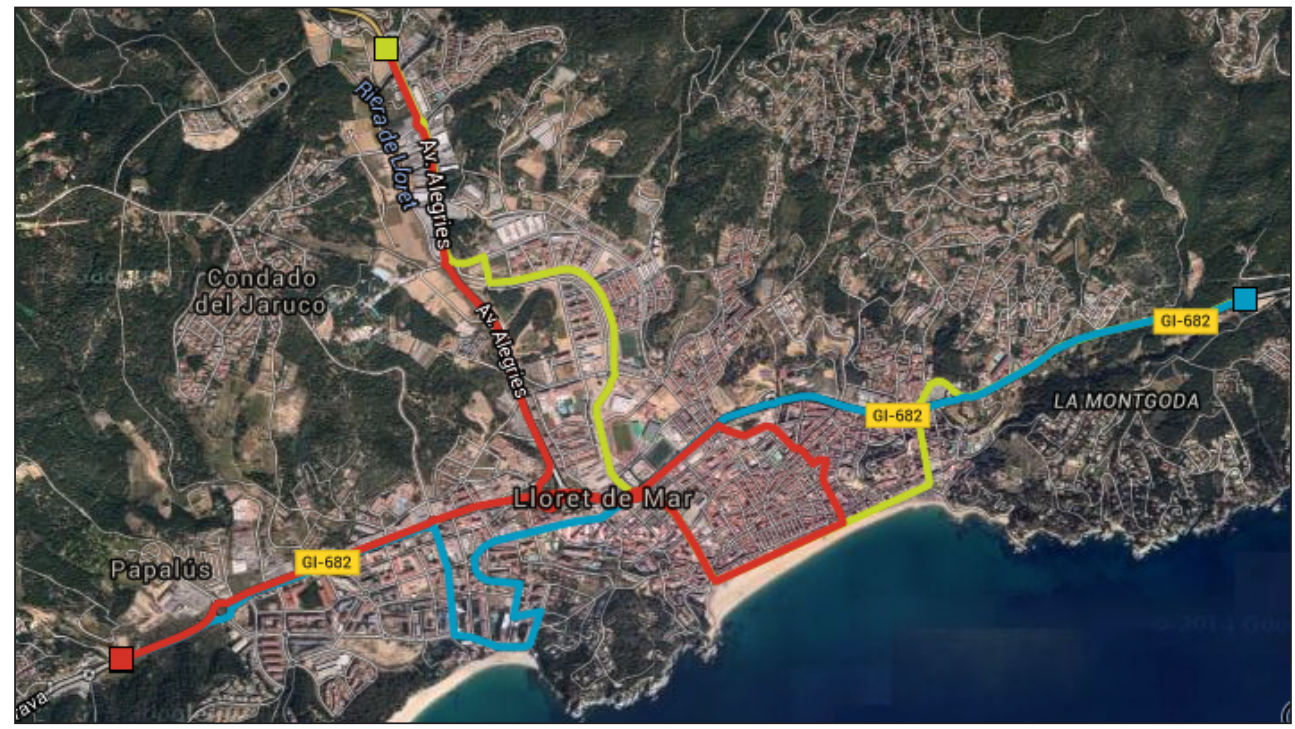

Fuente: elaboración propia a partir de Google Maps.

1 En color azul aparece el transecto 1, en color amarillo se señala el transecto 2 y en rojo esta el transecto 3. 


\section{Transecto 1}

Presenta una longitud total de $6,2 \mathrm{~km}$ y 21 puntos de toma de temperatura. El inicio se sitúa en la carretera GI-682 viniendo desde Blanes hacia Lloret, pocos metros después de la rotonda que sirve para desviarse hacia Santa Cristina. El final se ubica en la avenida Vila de Tossa, pocos metros antes del desvío hacia el sector de Cala Canyelles y Cala Gran.

El interés del transecto reside en su paso por el principal sector turístico de Fenals y parte de su sector residencial; así como por un extremo del barrio de la Riera, ya que no puede atravesarse de forma más céntrica debido a la peatonalización de parte de sus calles. También pasa por la avenida vila de Blanes y avenida vila de Tossa, la vía más transitada de la ciudad pues es una vía de redistribución del tránsito, además de ejercer como "cinturón de ronda" ya que une dos de las tres vías de entrada de la ciudad. Pasa, asimismo, por un punto neurálgico para el tráfico de la ciudad como es la gran rotonda cercana a la estación de autobuses. Además, se incluyen diferentes aéreas que suponen interrupciones en la edificación como el campo de futbol, dos grandes superficies de aparcamiento privado (una para coches y otra para autocares), y el sector de Can Xardó.

\section{Transecto 2}

El segundo transecto es el más corto, con una longitud de 5,6km y 21 puntos de toma de temperatura. Su inicio coincide con el final del transecto 1 en la avenida vila de Tossa, mientras que su final se ubica en la carretera C-63, dirección Vidreres, poco antes del desvío hacía Mas Romeu Residencial.

El interés del transecto radica, principalmente, por su paso por el frente marítimo de la ciudad, el principal centro de ocio nocturno y el barrio de El Rieral, el sector donde se ha dado el más reciente crecimiento urbano de la ciudad y cuya función es residencial. Además, también es interesante el paso por la única zona con características industriales de Lloret y poder comparar el comportamiento de dos importantes vías de entrada y salida de la ciudad como son las avenidas vila de Tossa y Vidreres.

\section{Transecto 3}

Este es el que se considera el más significativo, con una longitud total de $7,1 \mathrm{~km}$ y un total de 29 puntos de toma de temperaturas. El inicio se sitúa en la C-63, dirección Lloret, mientras que el final se localiza en la carretera GI-682 dirección Blanes, pocos metros de la rotonda que sirve para desviarse hacia Santa Cristina.

La mayor importancia de este transecto radica en que atraviesa por parte del sector más densamente edificado de la ciudad. Además también discurre por parte del frente marítimo y por la avenida Just i Marlès, sectores caracterizados por la importante actividad turística que presentan en verano (souvenirs, hoteles, bares, restaurantes y discotecas). Todo esto permitirá observar el comportamiento térmico diferencial, o no, entre distintos sectores y entre verano e invierno.

\section{RESULTADOS}

Las campañas de toma de temperaturas se desarrollaron en verano y en invierno, desde julio de 2010 a marzo de 2014. Así pues, consta de cuatro campañas estivales y cuatro 
campañas invernales. En total, se efectuaron 160 jornadas de mediciones, 80 estivales y 80 invernales, recopilando 11.360 valores térmicos y habiendo recorrido un total de $3.024 \mathrm{~km}$ en los transectos seguidos.

\section{IV.1. Campañas estivales}

Las cuatro campañas estivales fueron efectuadas en julio del 2010, junio y julio del 2011, julio del 2012, y junio y julio del 2013, con un de 5.680 valores térmicos recogidos. A continuación se presenta las intensidades registradas, el perfil térmico promedio del transecto 3 y la configuración media de la isla de calor estival.

\section{IV.1.1. Intensidad}

La máxima intensidad estival se registró durante la primera campaña de toma de temperaturas el día 15 de julio de 2010 , con un valor de $6,6^{\circ} \mathrm{C}$. La mínima intensidad se produce durante la segunda campaña, el día 12 de julio del 2011 y presenta un valor de $1,7^{\circ} \mathrm{C}$ (figura 5).

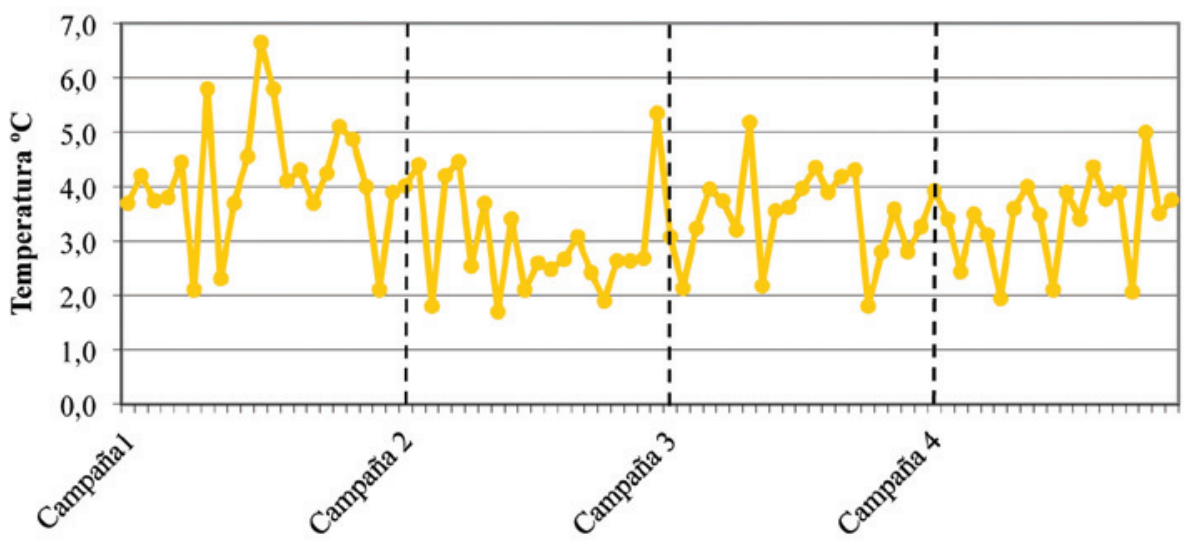

Fuente: elaboración propia.

Resulta destacable observar cómo en ninguno de los 80 días analizados durante las cuatro campañas estivales la intensidad de la isla de calor fue inferior a $\operatorname{los} 1,5^{\circ} \mathrm{C}$, por lo que se demuestra que la generación del fenómeno en la ciudad es recurrente durante la época estival.

Respecto al valor promedio de intensidad que registra la isla de calor durante las campañas estivales, éste se sitúa en $3,5^{\circ} \mathrm{C}$. La primera campaña es la que presenta un promedio de intensidades más elevado $\left(4,1^{\circ} \mathrm{C}\right)$, mientras que la segunda campaña $\left(3^{\circ} \mathrm{C}\right)$ es la que muestra un promedio más bajo. 


\section{IV.1.2. Configuración y localización del máximo térmico}

El mapa térmico estival de la isla de calor presenta un pequeño núcleo situado en el paseo Agustí i Font definido por una isoterma de $25^{\circ} \mathrm{C}$ (figura 6). Alrededor de este centro térmico, por el frente litoral y hacia el interior de la ciudad, aparece la isoterma de $24^{\circ} \mathrm{C}$ sobre parte de la superficie de los barrios del Nucli Antic y de la Riera.

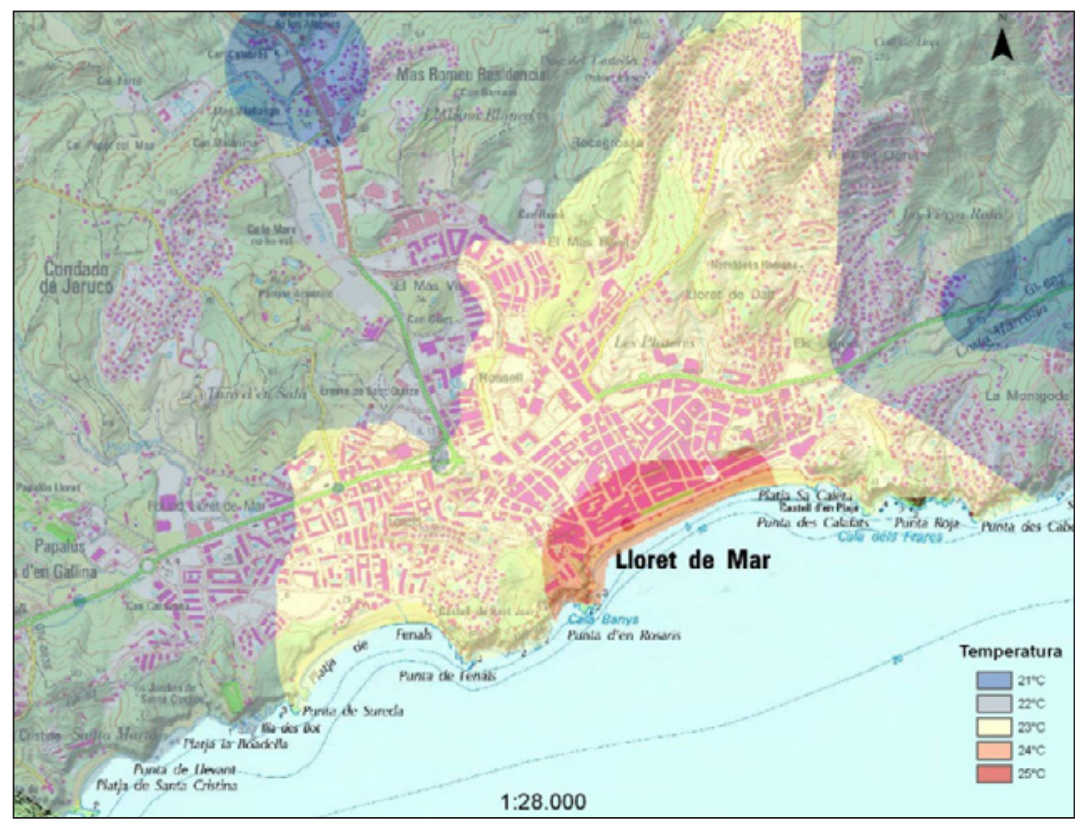

Base cartográfica del Instituto Geográfico Nacional de España.

El resto de trama urbana, ocupando la mayor extensión de ciudad, se encuentra bajo la influencia de los $23^{\circ} \mathrm{C}$. Aquí se encuentra la mayor parte de los barrios del Nucli Antic, de la Riera, de Can Sabata y de Fenals, además de los barrios de El Moli, Can Carbó y dels Pescadors.

Los sectores urbanos periféricos de la ciudad como el polígono industrial, el barrio de El Rieral, el barrio de Puigventós y el sector occidental de Fenals, así como parte de Can Sabata aparecen bajo la isoterma de $22^{\circ} \mathrm{C}$. Por último, cabe indicar como los sectores más fríos aparecen fuera de la ciudad siguiendo las vías de salida hacia Vidreres y Tossa de Mar. En estas áreas se observan isotermas de $21^{\circ} \mathrm{C}$. Cabe señalar que hacia Blanes, en la zona de cañizo entre las rotondas también se observa un valor entorno a los $21^{\circ} \mathrm{C}$.

Conviene señalar que esta configuración estival difiere un poco de las configuraciones observadas para cada campaña. La diferencia está, básicamente, en la extensión que presenta el centro térmico. En las campañas se observa un centro térmico con una mayor superficie 
que se extiende a lo largo de la playa de Lloret y hacia el interior del Nucli Antic, especialmente por la avenida Just i Marlés. Estos centros térmicos se asemejan más a la isoterma de $24^{\circ} \mathrm{C}$ que se observa en el mapa anterior.

\section{IV.1.3. Perfil térmico}

La diferencia máxima en el perfil estival muestra un valor de $3,4^{\circ} \mathrm{C}$. El máximo térmico del perfil presenta un valor de $25^{\circ} \mathrm{C}$ y se produce en el paseo Agustí i Font, en su tramo final que confluye con la avenida Just i Marlès. El mínimo térmico registra un valor de $21,6^{\circ} \mathrm{se}$ encuentra en la C-63, coincidiendo con el inicio del perfil (figura 7).

Figura 7

PERFIL TÉRMICO ESTIVAL DEL TRANSECTO 3

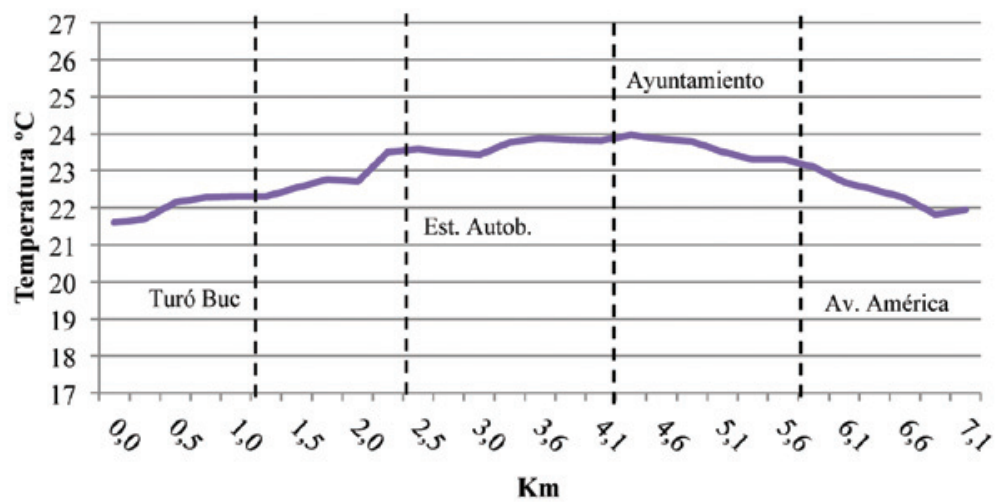

Fuente: elaboración propia.

El perfil se inicia registrando el mínimo térmico en la C-63. Al entrar en el polígono industrial de la avenida Vidreres, se aprecia como la temperatura comienza a ascender. No obstante, en la avenida Alegries, coincidiendo con la zona verde, ese ascenso se ve frenado al observarse una estabilización de la temperatura. Acto seguido, ya en Can Sabata, el ascenso térmico prosigue hasta llegar al puente sobre la avenida vila de Blanes, donde se muestra un ligero descenso. Una vez en la avenida Vila de Blanes, se produce un incremento térmico que llega hasta la Estación de Autobuses.

A partir de aquí, se produce un descenso de la temperatura a lo largo de la avenida de Blanes hasta llegar el Mercat Municipal. Este sector se caracteriza por la presencia de varios espacios libres o de baja densidad edificatoria como el campo de futbol, un aparcamiento, el centro comercial de la antigua plaza de toros o dos escuelas. Después, la temperatura se eleva a través del tramo de Rector Felip i Gibert y la Plaza París, hasta llegar a la calle Vall de Venècia, donde se observa el segundo valor más elevado del perfil. Posteriormente, se produce un leve descenso de la temperatura y una estabilización a lo largo de los paseos que conforman el frente marítimo, hasta que al final del paseo Agustí i Font se detecta el máximo térmico. 
En la avenida Just i Marlés se inicia un ligero descenso de la temperatura que después de dejar la rotonda de la Estación de Autobuses, ya en la avenida vila de Blanes se intensifica. En el tramo de la avenida justo antes de la rotonda de la avenida América se aprecia cómo la temperatura se estabiliza, para luego seguir desciendo hasta la salida de la ciudad. Una vez fuera del entorno urbano sigue el descenso a excepción del final del perfil, donde se eleva ligeramente.

\section{IV.2. Campañas invernales}

Las cuatro campañas invernales se efectuaron en enero y febrero del 2011; en enero y febrero de 2012; en enero, febrero y marzo de 2013; y en enero, febrero y marzo de 2014. Aquí también se analizan un total de 5.680 valores térmicos. Como en el caso de las campañas estivales se presentan las intensidades diarias, el perfil térmico promedio del transecto 3 y la configuración media de la isla de calor invernal.

\section{IV.2.1. Intensidad}

En la tercera campaña invernal es cuando se registra la máxima intensidad. El día 28 de enero de 2013 se obtiene una intensidad de $5,5^{\circ} \mathrm{C}$, mientras que la mínima intensidad es obtenida durante la primera campaña, el día 4 de febrero de 2011 con un valor de $0,9^{\circ} \mathrm{C}$, siendo el único día que se registra una intensidad inferior a $1^{\circ} \mathrm{C}$ (figura 8).

Así pues, durante los 80 días analizados en las cuatro campañas invernales, en tan solo una ocasión la intensidad ha mostrado un valor inferior a $1^{\circ} \mathrm{C}$. De este modo se observa que la aparición del fenómeno en la ciudad de Lloret durante el invierno es habitual.

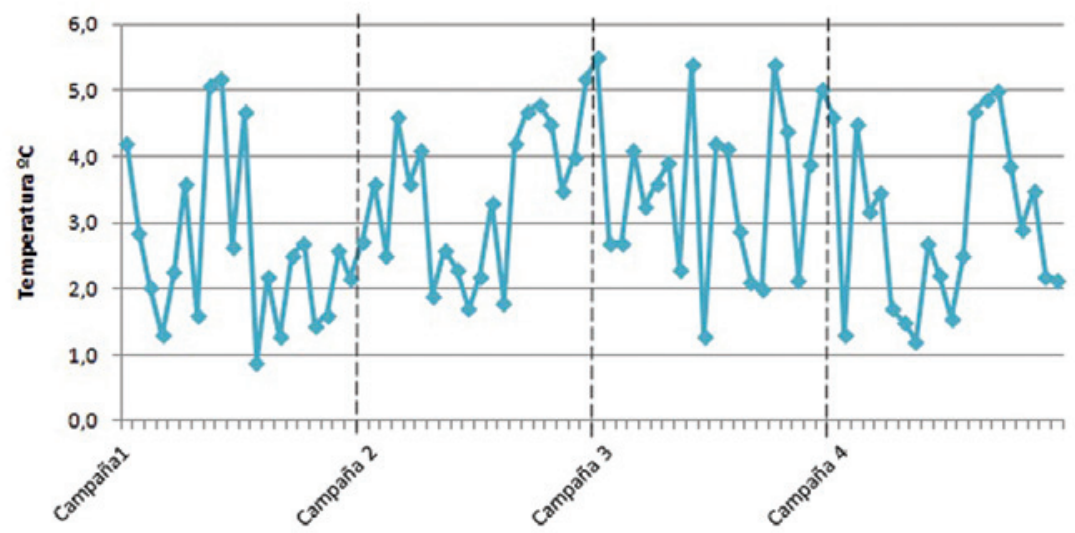

Fuente: elaboración propia. 
El valor promedio que presenta la intensidad de la isla de calor durante las campañas invernales, se sitúa en $3,1^{\circ} \mathrm{C}$. En la tercera campaña $\left(3,5^{\circ} \mathrm{C}\right)$ es donde se observa un promedio de intensidad más elevado, mientras que la primera $\left(2,7^{\circ} \mathrm{C}\right)$ es la que presenta un promedio más bajo.

\section{IV.2.2. Configuración y localización del máximo térmico}

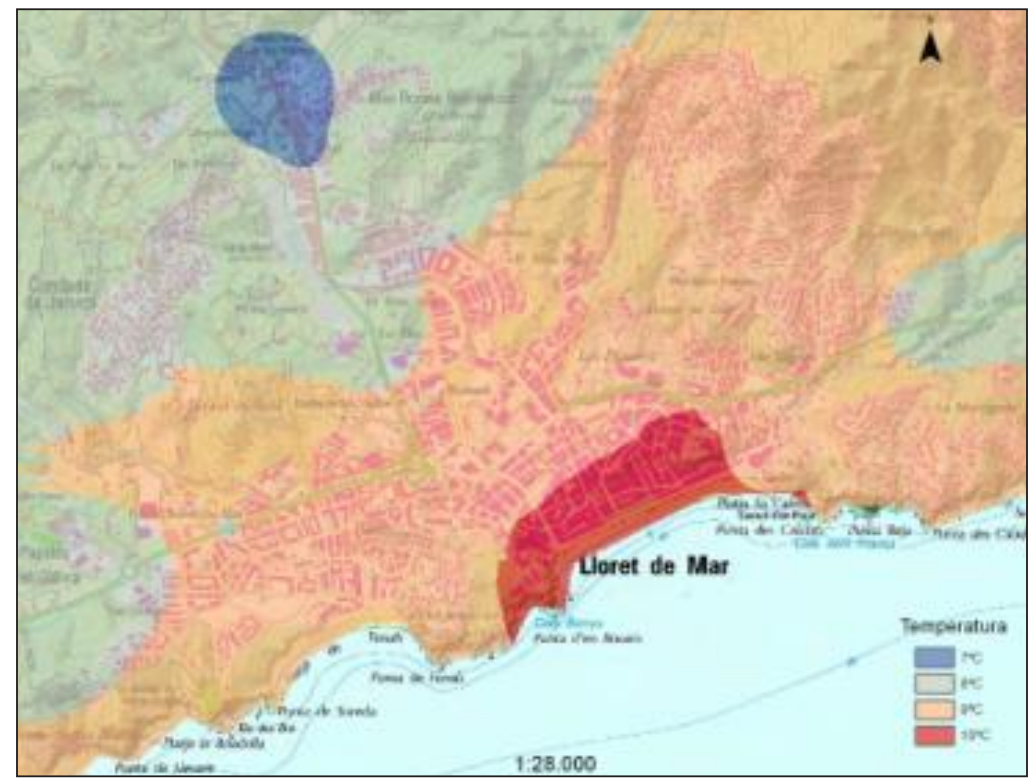

Fuente: Base cartográfica del Instituto Geográfico Nacional de España.

La configuración invernal de la isla de calor de Lloret de Mar muestra un máximo térmico con una mayor superficie que en el caso de cada campaña. Así se observa una isoterma de $10^{\circ} \mathrm{C}$, que marca el núcleo de la isla de calor, englobando la mayor parte del Nucli Antic y una parte del barrio de la Riera. En el caso de cada campaña, el centro térmico se encontraba localizado en el litoral ocupando una menor extensión del Nucli Antic (figura 9).

Alrededor del centro térmico, ocupando la mayor parte de extensión urbana aparece la isoterma de $9^{\circ} \mathrm{C}$. De este modo, tal como pasaba en cada campaña, se aprecia que los barrios residenciales de la ciudad, así como Fenals, presentan un comportamiento térmico similar.

El sector del barrio de El Rieral más cercano al polígono industrial, gran parte del polígono y el barrio de Puigventós se sitúan dentro de la isoterma de $\operatorname{los} 8^{\circ} \mathrm{C}$. De igual forma, el límite occidental de Fenals también está bajo dicha isoterma. Conviene señalar como el sector de la avenida vila de Blanes cercano al cámpig Santa Elena muestra una isoterma cerrada de $8^{\circ} \mathrm{C}$. Por último, la isoterma de $7^{\circ} \mathrm{C}$ aparece sobre la $\mathrm{C}-63$ y una parte del polígono industrial. 


\section{IV.2.3. Perfil térmico}

El perfil invernal muestra una diferencia máxima de $2,4^{\circ} \mathrm{C}$. El máximo térmico del perfil que presenta un valor de $10,3^{\circ} \mathrm{C}$, se produce en el tramo donde el paseo Camprodon coincide con el paseo Jacint Verdaguer y la calle Venecia con la calle de la Vila. El mínimo térmico se registra, como siempre, al inicio del perfil con un valor de $7,9^{\circ} \mathrm{C}$ (figura 10 ).

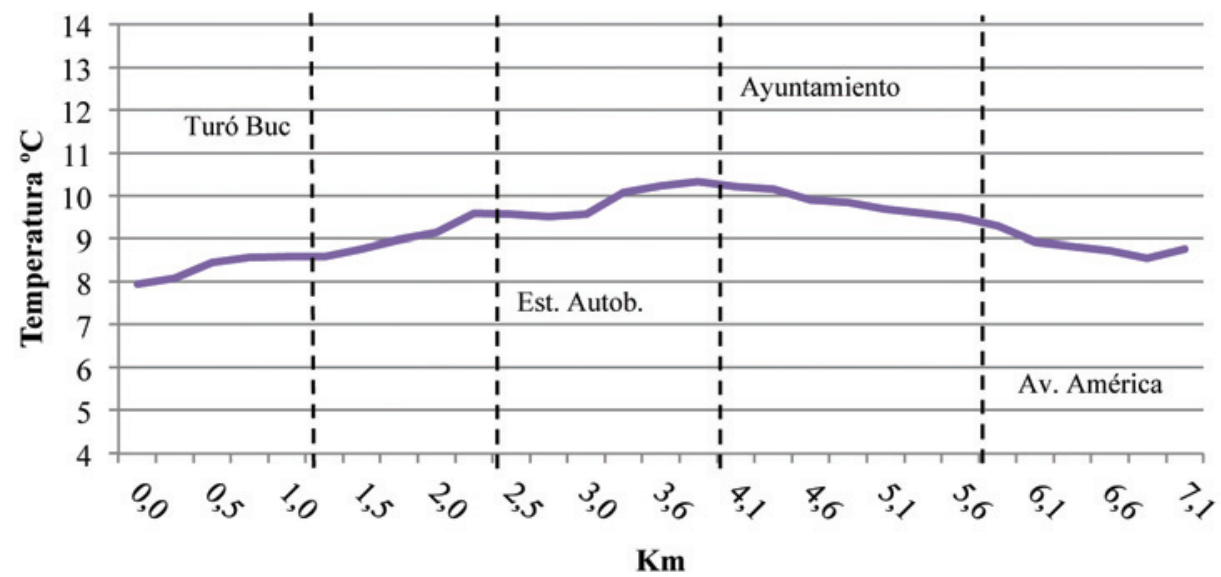

Fuente: elaboración propia.

El inicio del perfil viene marcado por registrar el mínimo térmico en la C-63. En el sector industrial se genera un ascenso de la temperatura que al llegar a la avenida Alegries se torna en estabilidad térmica hasta penetrar en el barrio de Can Sabata, donde se vuelve a elevar la temperatura. El incremento térmico dura hasta la Estación de Autobuses, después de la cual se observa un nuevo tramo de estabilidad de la temperatura a lo largo de la avenida vila de Blanes hasta llegar al Mercat Municipal, en la calle Rector Felip i Gibert.

Después del Mercat, la temperatura se eleva, especialmente en la plaza París, y se alcanza el máximo térmico en la calle Venecia con el paseo Camprodon. Posteriormente, la temperatura desciende, primero de forma leve en el frente marítimo, luego, en la avenida Just i Marlès, el descenso se muestra un poco más intenso. Al entrar en la avenida vila de Blanes se aprecia un descenso más acusado. El descenso continúa y llega casi al final de perfil. Una vez más, el final del perfil viene marcado por un leve ascenso de la temperatura.

\section{IV.3. Características generales de la isla de calor de Lloret de Mar}

La intensidad media del fenómeno de isla de calor en la ciudad presenta un valor de $3,3^{\circ} \mathrm{C}$. La intensidad estival $\left(3,5^{\circ} \mathrm{C}\right)$ se muestra más elevada que la intensidad invernal $\left(3,1^{\circ} \mathrm{C}\right)$. El promedio de todos los valores térmicos para cada uno de los puntos de observa- 
ción muestra el paseo Agustí i Font como el más cálido, mientras que el punto más frío se sitúa en la C-63 marcando el final del transecto 2 y el inicio del transecto 3. Destaca cómo el punto más cálido invernal y estival no coinciden, lo mismo sucede con el punto más frío invernal y estival, por lo que se observan diferencias entre las dos épocas del año.

En relación a la máxima intensidad que se recoge durante todas las campañas, ésta se produce en la primera campaña estival $\left(6,6^{\circ} \mathrm{C}\right)$, siendo $1,1^{\circ} \mathrm{C}$ mayor que la máxima intensidad invernal, obtenida en la tercera campaña invernal $\left(5,5^{\circ} \mathrm{C}\right)$. La intensidad mínima se registra durante la primera campaña invernal $\left(0,9^{\circ} \mathrm{C}\right)$ y es $0,8^{\circ} \mathrm{C}$ más fría que la intensidad mínima estival $\left(1,7^{\circ} \mathrm{C}\right)$, observada durante la segunda campaña.

La tabla 1 muestra las intensidades registradas durante los 160 días analizados clasificadas según la propuesta de Fernández (1995). A grandes rasgos, se observa como las intensidades moderadas (de $2^{\circ} \mathrm{C}$ a $4^{\circ} \mathrm{C}$ ) son las que más se producen ya que se dan en algo más de la mitad de los días analizados. También resultan destacables las jornadas que presentan una intensidad fuerte $\left(\mathrm{de} 4^{\circ} \mathrm{C}\right.$ a $6^{\circ} \mathrm{C}$ ), representando casi $1 / 3$ del total.

Tabla 1

CLASIFICACIÓN DE LA INTENSIDADES DE LA ISLADE CALOR

\begin{tabular}{|l|rr|rrrr|}
\hline Intensidades & \multicolumn{2}{c|}{ Verano } & \multicolumn{2}{c|}{ Invierno } & \multicolumn{3}{c|}{ Total } \\
\hline $0^{\circ} \mathrm{C} \mathrm{a} 2^{\circ} \mathrm{C}$ & 5 & $6,3 \%$ & 15 & $18,8 \%$ & 20 & $12,5 \%$ \\
$2^{\circ} \mathrm{C} \mathrm{a} 4^{\circ} \mathrm{C}$ & 50 & $61,3 \%$ & 40 & $50,0 \%$ & 89 & $55,6 \%$ \\
$4^{\circ} \mathrm{C} \mathrm{a} 6^{\circ} \mathrm{C}$ & 25 & $31,3 \%$ & 25 & $31,3 \%$ & 50 & $31,3 \%$ \\
$6^{\circ} \mathrm{C} \mathrm{y}+$ & 1 & $1,3 \%$ & 0 & $0,0 \%$ & 1 & $0,6 \%$ \\
\hline Totales & 80 & $100 \%$ & 80 & $100,0 \%$ & 160 & $100,0 \%$ \\
\hline
\end{tabular}

Fuente: elaboración propia.

La configuración general de la isla de calor de Lloret presenta el máximo térmico extendiéndose por el frente litoral. Una isoterma de $17^{\circ} \mathrm{C}$ define éste y engloba la mayor parte del Nucli Antic y la parte del barrio de la Riera más próxima al mar. Así pues, se observa cómo en las diferentes campañas, invernales y estivales, el centro de la isla de calor se localiza en el litoral (figura 11).

Alrededor del centro térmico, ocupando la mayor parte de extensión urbana se aprecia la isoterma de $16^{\circ} \mathrm{C}$. Esto muestra que los barrios residenciales de la ciudad, así como la mayor parte de Fenals, presentan un comportamiento térmico similar. Algo que se ha observado en las configuraciones promedio de cada campaña, ya sean estivales o invernales.

Los sectores urbanos más frescos se encuentran en la zona del barrio de El Rieral más cercano al polígono industrial y en gran parte de éste que están bajo la influencia de la isoterma de $\operatorname{los} 15^{\circ} \mathrm{C}$. De igual forma, el límite occidental de Fenals también está bajo dicha isoterma. Finalmente, la isoterma de $14^{\circ} \mathrm{C}$ determina el valor más frío que se aprecia sobre la C-63.

Lo más destacable de la configuración espacial del fenómeno de la isla de calor es la extensión del centro térmico y cómo ocupa una mayor o menor extensión en las distintas campañas. Por lo general, en verano aparece un centro térmico más extenso que penetra 
hacia el interior del Nucli Antic y hacia el sector turístico, mientras que en invierno el centro térmico muestra una extensión menor ubicándose de forma más localizada en el paseo Jacint Verdaguer y el sector del Camprodon próximo a éste. De este modo se aprecia que el barrio del Nucli Antic tiene un comportamiento térmico diferente al resto de la ciudad al localizarse sobre éste el centro térmico y donde la actividad turística parece afectar a que el núcleo presente una mayor extensión estival.

Otro hecho destacable reside en cómo, en general, las vías de salida de la ciudad parecen canalizar aire rural, más fresco, hacia la ciudad, especialmente la salida hacia Vidreres. No obstante, hay que mencionar que en ocasiones el calor urbano avanza hacia el entorno rural hacia Tossa o Blanes. Por último, queda por indicar la presencia de algunos puntos de frescor que suelen aparecer en torno al sector de las escuelas de la calle Rector Felip i Gibert y del campo de fútbol de la avenida vila de Blanes.

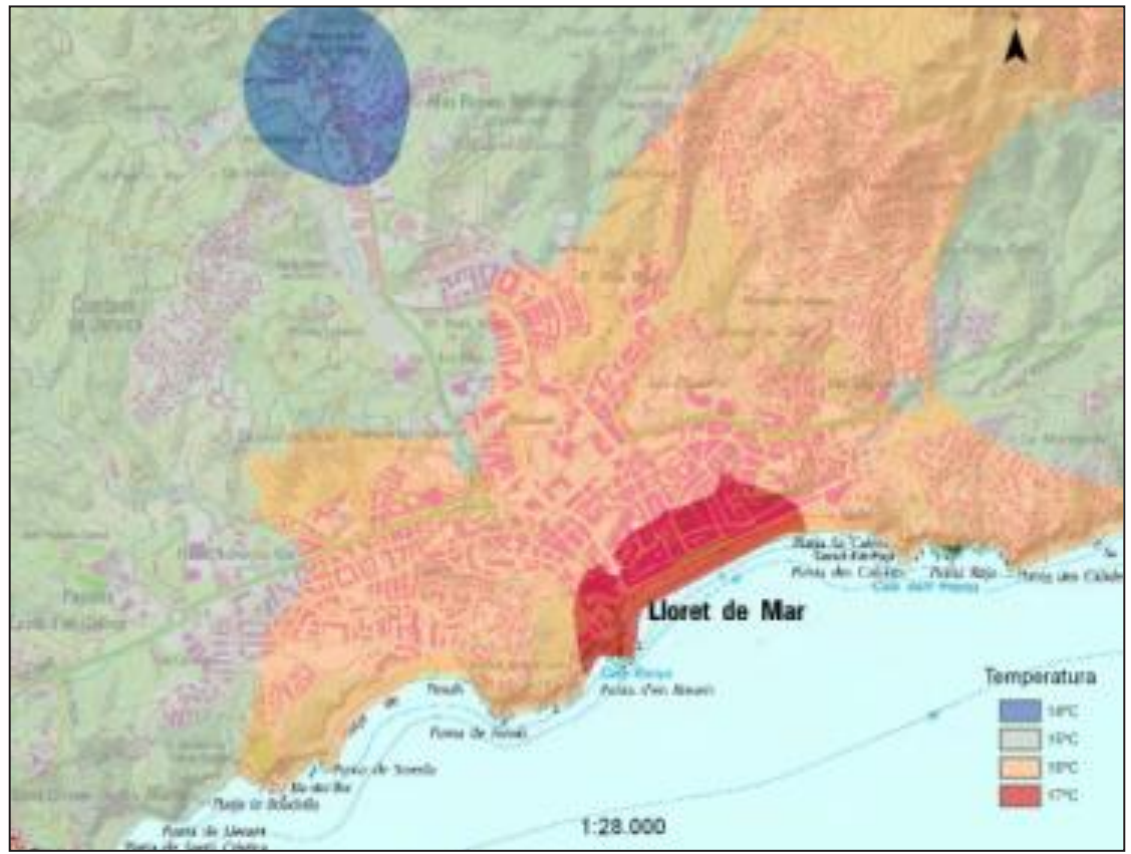

Base cartográfica del Instituto Geográfico Nacional de España,

La diferencia máxima del perfil térmico promedio muestra un valor de $2,3^{\circ} \mathrm{C}$. El máximo térmico se observa en el paseo Camprodon delante del Museu del Mar, mientras que el mínimo térmico se sitúa en el inicio del transecto. Cabe recordar que éste máximo térmico coincide con el perfil invernal, mientras que en el perfil estival se encuentra en el paseo Agustí i Font. Mientras que los mínimos térmicos coinciden con el inicio del perfil en la C-63 (figura 12). 


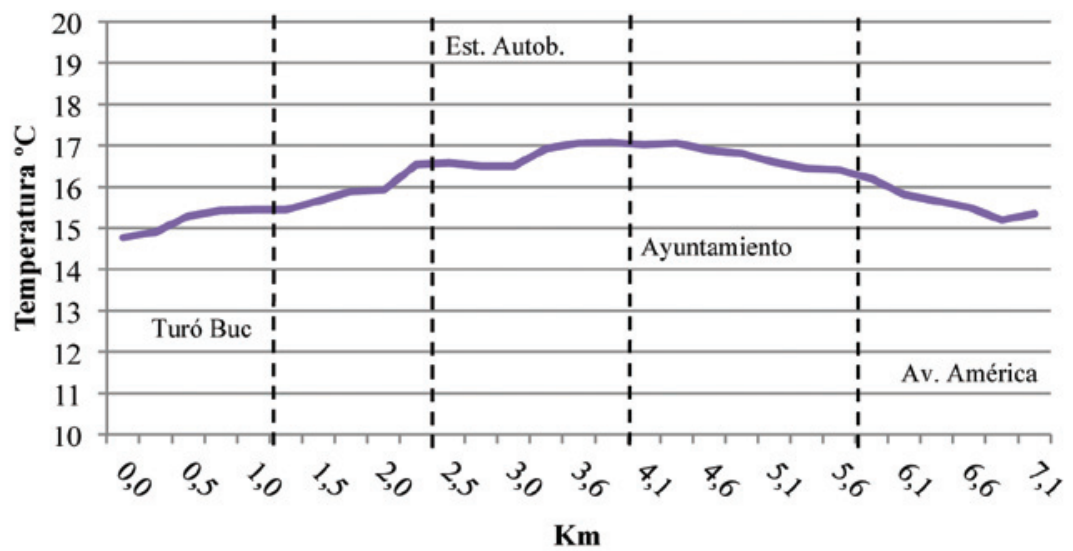

Fuente: elaboración propia.

El perfil registra su mínimo térmico en el inicio. A partir de éste la temperatura inicia un incremento que llega hasta después de la Estación de Autobuses. No obstante, se aprecian dos tramos de cierta estabilidad térmica en la avenida Alegries, el primero entre el polígono industrial y Can Sabata y, el segundo, en el sector posterior a Can Sabata antes de entrar a la avenida vila de Blanes. Una vez pasada la Estación de Autobuses la temperatura desciende levemente en el sector del campo de futbol, el centro comercial de la antigua plaza de toros y el Mercat Municipal. Posteriormente, se incrementa la temperatura hasta entrar en la calle Vall de Venècia donde se observa un tramo de cierta estabilidad térmica seguido del máximo térmico en el Museo del Mar. Después de un leve descenso de la temperatura en el paseo de Jacint Verdaguer se dibuja un leve ascenso en el Ayuntamiento, seguido de cierta estabilidad en el paseo Agustí i Font. En la avenida Just i Marlès se inicia un descenso térmico que se extiende a lo largo del perfil hasta llegar casi al final. Al final del perfil se muestra un leve ascenso térmico.

A grandes rasgos, conviene señalar los siguientes aspectos de los perfiles térmicos:

- Espacios abiertos que introducen cierto frescor. A lo largo de los perfiles analizados se puede apreciar que varios sectores urbanos, caracterizados por una poca densidad edificatoria, introducen descensos térmicos o frenan el incremento térmico que se venía sucediendo. Sectores que en muchas ocasiones aparecen cartografiados en los mapas de isotermas. Los sectores más significativos están en el tramo de la avenida Alegries que transcurre próximo al área verde del Turó d'en Buc y el tramo de la avenida vila de Blanes, justo después de la Estación de Autobuses, que llega hasta el Mercat Municipal. De forma menos significativa, también debe mencionarse el tramo de después de Can Sabata que es el acceso a la avenida vila de Blanes camino a la Estación de Autobuses. 
- El diferente comportamiento del máximo térmico según sea invierno o verano, algo que también se ha visto en el mapa de isotermas. En las campañas estivales los máximos térmicos se encuentran sobre el paseo Agustí i Font, próximos a la avenida Just i Marlès. En cambio, en invierno los máximos térmicos se ubican en el paseo Camprodon, cercano a la calle Vall de Venècia. También debe mencionarse que durante el verano, en el frente litoral, se aprecia una mayor estabilidad térmica, mientras que en invierno suele observarse un suave descenso de la temperatura. Estos diferentes comportamientos según la estación del año son susceptibles de ser provocados por el turismo que genera una mayor actividad humana en el frente litoral y alrededor de la avenida Just i Marlès.

- El incremento térmico al final del perfil. Resulta curioso cómo el final del perfil siempre suele mostrar un pequeño repunte de la temperatura. Ésto puede venir generado por las características que presenta este tramo final, donde la salida de la ciudad significa el fin del límite de velocidad de $50 \mathrm{~km} / \mathrm{h}$ y genera una mayor aceleración de los conductores que se encuentran con una pendiente y que acaba significando una mayor generación de gases contaminantes.

\section{CONCLUSIONES}

El análisis de las mediciones efectuadas en las distintas campañas pone de manifiesto un claro contraste térmico entre los sectores urbanos y los sectores rurales del municipio de Lloret de Mar dando origen a una isla de calor urbana en la ciudad. La intensidad del fenómeno llega a $3,3^{\circ} \mathrm{C}$ de media, pudiendo alcanzar valores máximos importantes de hasta $6,6^{\circ} \mathrm{C}$.

La configuración espacial de la isla de calor muestra cómo la parte central de la ciudad, Nucli Antic, presenta un comportamiento térmico más cálido que los barrios residenciales próximos a éste. El sector de Fenals presenta el mismo comportamiento que los otros barrios residenciales con los que comparten varias características morfológicas urbanas. Los sectores urbanos periféricos del norte de la ciudad, El Rieral y el área industrial de la avenida Vidreres, se muestran como los sectores más fríos de la ciudad. Sobre éstos parece que el aire rural penetra hacia la ciudad. En ocasiones se detectan puntos de frescor, delimitados por isotermas cerradas de menor valor que el territorio donde se inscriben, que coinciden con sectores con menor densidad edificatoria (Campo de Futbol de la avenida vila de Blanes y las escuelas de la calle Rector Felip i Gibert).

El núcleo de la isla de calor se encuentra situado sobre el litoral de la ciudad, extendiéndose por los paseos que se suceden en la playa de Lloret y penetrando hacia el interior del barrio del Nucli Antic.

Finalmente, la comparación de las campañas estivales e invernales deja ver cómo se producen unas diferencias entre estas dos épocas del año. Así pues, en verano: se registra una intensidad media algo más elevada $\left(3,5^{\circ} \mathrm{C}\right.$ frente a los $\left.3,1^{\circ} \mathrm{C}\right)$; el núcleo del fenómeno presenta una mayor superficie al desparramarse por el sector turístico alrededor de la avenida Just i Malés; y el perfil térmico muestra cómo el máximo térmico se desplaza del paseo Camprodon al paseo Agustí i Font, en el sector próximo a la avenida Just i Marlés.

La actividad turística parece que podría ser la causante, especialmente en la configuración espacial, de estas diferencias. Abtualeb et al. (2014) en su investigación en la ciudad de Ale- 
jandría señalan algo semejante ya que en verano registran una mayor intensidad los sectores de la ciudad más repletos por los visitantes. Sin olvidar que las condiciones meteorológicas es un factor determinante en los fenómenos de isla de calor y, tal como señaló Oke (1982), en verano parecen desarrollarse mejor dicho fenómeno, cuando se produce una mayor actividad turística y un incremento del calor antrópico que ésta genera en la ciudad de Lloret. Calor antrópico originado por el importante crecimiento de la población que registra la ciudad debido a la llegada de turistas. Este crecimiento poblacional conlleva la puesta en funcionamiento de unidades habitacionales (hoteles, hostales y segundas residencias) y locales (bares, restaurantes, tiendas, etc.), cerrados en invierno, y la presencia de un flujo de tráfico más denso (autobuses con mayor frecuencia y números de líneas en funcionamiento; autocares turísticos que transportan desde el origen del turista o para desplazamientos internos; mayor presencia de camiones de reparto debido al mayor consumo; y más coches que traen los nuevos habitantes). Por último cabe recordar cómo un buen número de trabajos relacionan la magnitud de la ICU con el tamaño de la ciudad, por ejemplo Oke (1973), sugiriendo que el incremento de temperaturas de algunas ciudades estaba en función del crecimiento de la población. En verano, en la ciudad se produce un crecimiento de la población en algo más de 32.500 personas $^{2}$, por lo que se pasa de una población de 40.803 habitantes en invierno a más de 73.000 habitantes en verano. Esto puede influir en la mayor intensidad estival.

\section{BIBLIOGRAFÍA}

ABTUALEB, K.; ADELINE, N.; AHMED, F.; AHMED, M.H.; ELKAFRAWY, S.B.; ARAFAT, S.M. y DRWISH, A. (2014): "Investigation of urban heat island usig Landsat data". En Proceedings of the 10th International Conference of African Association of Remote Sensing of the Environment, 27-31 October 2014, Johannesburg.

ACERO, J.A.; ARRIZALABAGA, J.; KUPSKI, S. y KATZSCHNER, L. (2013): "Urban heat island in a coastal urban area in northern Spain", Theoretic and Applied Climatology, vol. 113, pp. 137-154.

ALONSO, M.S., LABAJO, J.L. y FIDALGO, M.R. (2003): "Characteristics of the urban heat island in the city of Salamanca, Spain”, Atmósfera, vol. 16 (3), pp. 137-148.

ARNFIELD, A.J. (2003): "Two decades of urban climate research: a review of turbulence, exchanges of energy and water, and the urban heat island", International Journal of Climatology, $\mathrm{n}^{\circ} 23$, pp. 1-26.

BELLO FUENTES, V. (1994): "La isla de calor y los usos del suelo en Guadalajara", Serie Geográfica, Vol. 4, pp. 83-97.

BELLO FUENTES, V. (1995): "La isla de calor nocturna y los usos del suelo en Alcalá de Henares", Anales de la Geografía de la Universidad Complutense, n 15, pp. 119-130.

BRUNET, M. (1992): "La magnitud y fluctuaciones de la isla de calor de Tarragona", Tarraco, $\mathrm{n}^{\circ}$ 7, pp. 19-29.

2 La encuesta de Ocupación Hotelera estima que para el año 2013 en la ciudad de Lloret de Mar habían 32.502 plazas hoteleras y se produjo la entrada de 1.067 .802 viajeros, de las que 162.826 fueron en el mes de julio y 178.635 en el mes de agosto. A estas cifras hay que sumarle los datos de las plazas en otros tipos de alojamientos (camping, alquiler...) y segunda residencia. 
CASTILlo CASTÁN, V. (2006): "El calentamiento urbano: la isla de calor en Monzón (Huesca)", Lucas Mallada, no 13, pp. 47-90.

CUADRAT PRATS, J.M.; DE LA RIVA FERNANDEZ, J.; LOPEZ MARTIN, F. y MARTI EZPELETA, A. (1993): "Ciudad y medio ambiente: la isla de calor de Teruel", Geographicalia, no 30 , pp. 113-123.

DE LA MORENA CARRETERO, B.A. (2010): "Estudio de la isla de calor urbana en el área metropolitana de Sevilla", en CONAMA $10^{\circ}$ Congreso Nacional del Medio Ambiente, Madrid.

DOMÈNECH MONER, J. (1982): Cinquanta anys d'esforç turístics a Lloret de Mar. Servei Municipal de Publicacions. Lloret de Mar, 152 pp.

DOMÍNGUEZ BASCÓN, P. (2002): Clima regional y microclimas urbanos en la provincia de Córdoba. Servicios de publicaciones de la Universidad de Córdoba. Córdoba, $110 \mathrm{pp}$.

DORTA, P.; MARZOL, V.; RODRIGUEZ, J. (1992) "Estudio del clima urbano de una ciudad del litoral. El caso de S/C de Tenerife (I. Canarias)". VI Trobadas Cientifiques de la Mediterrania: Energía, Medi Ambient i Edificació. Menorca. Ins. d'Est. Catalans.

FERNÁNDEZ GARCÍA, F. (1995): Manual de climatología aplicada: Clima, medioambiente y planificación. Ed. Síntesis. Espacios y Sociedades. Madrid, 285 pp.

FERNÁNDEZ GARCÍA, F.; GALÁN GALLEGO, E. y CAÑADA TORRECILLA, R. (Coords.) (1998): Clima y ambiente urbano en ciudades ibéricas e iberoamericanas. Editorial Parteluz. Madrid, 606 pp.

FERNÁNDEZ GARCÍA, F. (2009): “Ciudad y cambio climático: aspectos generales y aplicación al área metropolitana de Madrid”, Investigaciones Geográficas, no 49, pp. 51-63.

LÓPEZ GÓMEZ, A.; FERNÁNDEZ, F.; ARROYO, F.; MARTÍN VIDE, J.; CUADRAT, J.M. (1993): El clima de las ciudades españolas. Editorial Cátedra. Madrid, 268 pp.

MARTÍNEZ MARTÍNEZ, J. (2014): "Estudio de la isla de calor de la ciudad de Alicante", Investigaciones Geográficas, nº 62, pp. 83-99.

MONTÁVEZ, J.P., RODRÍGUEZ, A. y JIMÉNEZ, J.I. (2000): “A study of the urban heat island of Granada", International Journal of Climatology, $\mathrm{n}^{\circ}$ 20, pp. 899-911.

MORENO GARCÍA, M.C. (1993): Estudio del clima urbano de Barcelona: la «isla de calor». Editorial Oikos-tau. Barcelona, $193 \mathrm{pp}$.

MORENO GARCÍA, M.C. (1999): Climatología Urbana. Edicions Universitat de Barcelona. Barcelona, $71 \mathrm{pp}$.

OKE, T.R. (1973): "City size and the urban heat island", Atmospheric Environment, vol. 7, pp. 769-779.

OKE, T.R. (1982): “The Energetic Basis of the Urban Heat Island”, Quarterly Journal of the Royal Meteorological Society, vol. 108, pp. 1-24.

ORTIGOSA IZQUIERDO, I., SOBRÓN GARCÍA, I. y GÓMEZ VILLAR, A. (1998): “El microclima urbano de Logroño en invierno: un modelo espacial de comportamiento higrotérmico", Cuadernos de Investigación Geográfica, n 24, pp. 89-106.

QUEREDA SALA, J.; MONTÓN CHIVA, E. y ESCRIG BARBERÁ, J. (2007): “Un análisis experimental del efecto urbano sobre las temperaturas", Investigaciones Geográficas, $\mathrm{n}^{\circ}$ 47, pp. 5-17. 
RAMIRO ROCA, E. (2006): "Una isla que se mueve”, Didáctica Geográfica, $2^{\circ}$ época, n 8, pp. 33-55.

RUIZ-FLAÑO, P., ROMERO MARTÍN, L., MÁYER SUÁREZ, P., HERNÁNDEZ CORDERO, A. (2008): "La isla de calor en Las Palmas de Gran Canaria: intensidad, distribución y factores condicionantes", Boletín de la Asociación de Geógrafos españoles, $\mathrm{n}^{\circ}$ 47, pp. 157-173.

SERRA PARDO, J.A. (2007): "Estudio de la isla de calor de la ciudad de Ibiza", Investigaciones Geográficas, n ${ }^{\circ} 4$, pp. 55-73.

TORNERO, J., PÉREZ CUEVA, A. y GÓMEZ LOPERA, F. (2006): “Ciudad y confort ambiental: estado de la cuestión y aportaciones recientes", Cuadernos de Geografía, no 80, pp. 147-182.

VICENTE SERRANO, S.M., CUADRAT PRATS, J.M., SANZ SÁNCHEZ, M.A (2005): "Spatial patterns of the urban heat island in Zaragoza (Spain)", Climate Research, Vol. 30 (1), pp. 61-69. 
